\title{
Critiquing Barrett's How emotions are made
}

\author{
Ho Manh Tung \\ Ritsumeikan Asia Pacific University, \\ Beppu, Oita, Japan
}

October 13, 2020

\section{A brief introduction}

In recent years, there has been a growing trend in the tech and business communities to produce, sell, and capitalize on smart algorithms that are capable of reading, tracking, sensing, classifying human emotions - technologies that are often referred to as emotional artificial intelligence (emotional AI) or empathic media (McStay, 2018). In How Emotions Are Made: The Secret Life of the Brain, neuroscientist Lisa Feldman Barrett (2017) presents a critique that this trend is largely based on the essentialist view of emotions, which she called the myth of universal emotions. The essentialist view of emotion, held by many thinkers throughout history, from Plato to the modern-day psychologist Paul Ekman (1999), posits that there is a universal underlying causal mechanism for each emotion. Each emotion, the way it feels subjectively, and how we express them have distinct universal features shared across culture.

Drawing the most recent empirical and theoretical advances in brain science, Barret (2017) presents a very different counterintuitive account of emotion, which she called the "theory of constructed emotion." The constructed theory of emotion takes emotion as abstract categories formed through our interactions with the physical and social world. This theory is based on two components: the "predictive brain" view that the brain is stored inside a dark cage, 
our skull, and only has electrical signals from the body to figure out the external environment; and the "allostasis" view that the brain's function is to move the body while optimally allocate metabolic resources based on its prediction of the external environment. As such, emotions are constructed as a means for the brain to move the body, a set of algorithms of sort, which help us navigate the physical and social world. In this view, for the brain to construct emotions such as fear, anger, happiness, etc., it must learn about these abstract categories of emotion by inferring and updating them through input from the physical and social environment.

\section{Merits of Barrett (2017)'s account of emotion}

There are many merits to this account of emotion. First, it is based on very important theories that emerged in brain science over the last decade, backed by successfully making predictions and explaining human cognition and behaviors (Sterling, 2012). In many ways, it has provided new modes of scientific explanation (Deustch, 2011) of our emotional lives.

Second, Barrett's (2017) account of emotion is a valuable challenge raised to the tech and business communities that have been taken over by the view that as long as big data empower their AI, it will correctly read and classify human emotions. There have been many high-profile studies that confirm the fact that accuracy in inferring emotional expressions is contextdependent (Benitez-Quiroz, Srinivasan, \& Martinez, 2018; Chen \& Whitney, 2019). Indeed, the tacit dimension (Polanyi, 2009) of cultural knowledge about emotion is indeed something that has been underappreciated, to say the least, by people in the tech and AI-startups communities.

Third, unlike the pure social constructionist view of emotion, which could easily lead to cultural relativism about emotion, Barrett's theory takes into account the evolutionary pressure that shapes our emotional lives. This enables scientists to strive for an empirical and scientific 
understanding of the cultural variations for each emotion category: anger, shame, etc. The evolutionary aspect of Barret (2017)'s theory of constructed emotion also allows deduction from the first principles whether the socio-cultural evolutionary pressure is stronger or weaker than the natural evolutionary pressure.

\section{Some critiques}

However, there are many areas where Barret (2017) can improve upon. Although her book emphasizes the socio-cultural reality of emotions, she has not dealt properly with how people unlearn and relearn their emotional concepts when encountering new, possibly conflicting cultures. This point has been mentioned in a review of Jurist (2019). This issue of "acculturation" (Vuong \& Napier, 2015) and "cultural additivity" (Vuong et al., 2018; Vuong et al., 2020) is of great significance and should be dealt with more seriously. This is because emotional lives are at risk of being hijacked by the emergence of many cultural values of many new sub-cultures caused by the hyperconnectivity of Web 2.0. and globalization.

Second, although Barrett's (2017) critique of the current trend in producing and marketing emotional AI technologies is of great value, there are values to be found in letting the machines tell us about regularities in our emotional and bodily sensations patterns. Clearly, there are dangers in overclaiming the capacity of these new technologies, and there are even more dangers in letting them decide how we should feel and act about ourselves and others. Yet, as our lives are now inevitably permeated with technologies that notify us about our biometrics, taking advantage of this situation is our choice and responsibility.

Third, it is arguable that Barrett could make more of her theory by either devising alternative basic categories of emotions that might be applicable to the tech communities or 
capturing the algorithmic aspects of natural emotional recognition within her framework. Instead of the so-called universal basic emotions proposed by Paul Ekman, Barrett (2017) advocates for the education of what she called "emotional granularity," i.e., people should learn all the different shades of, for example, anger: hostility, rage, aggravation, frustration, etc. Being educated about and able to categorize as well as express all the granularities are clearly an inspiring and noble goal for people and society. Yet, this goal seems too tall an order for the tech communities, which need well-defined structured emotional categories to feed into their algorithms.

Currently, many AI-startup companies build their algorithms and databases (McStay, 2018; Mohammad, \& Turney, 2013) referring to focus on the eight basic emotions proposed by Paul Ekman (1999): anger, fear, sadness, disgust, surprise, anticipation, trust, and joy. As a practical solution, I would add to this list of awe, curiosity, doubt, confusion, shame, stress, embarrassment, indifference (cynicism). These are cross-cultural basic abstract categories of our emotional lives that have could be explained using the evolutionary and predictive-brain aspects of

To further refine Barret's theory, I think there are two research projects that can be started here. First, it seems to me that a detailed mapping of emotion words or concepts (anger, happiness, sadness, shame, disgust, etc.) and their cultural variations in terms of felt, subjective experiences and facial expressions, and their triggers (social injustice, revenge, social connection, creative discovery, etc.) are of great value. This research project seems difficult, but it can start with a systematic review or a meta-analysis of research on emotions. The second project can be about the current evolutionary pressures exerted by our new technologies: social media, emotional AI, etc. With advance in sensors and computations, I believe there are many readily 
available data to test the predictions generated by Barrett (2017)'s constructionist account of emotion. The most important thing in this process is to operate with the spirit of scientific humility (Vuong, 2020).

\section{References}

Barrett, L. F. (2017). How emotions are made: The secret life of the brain. London: Houghton Mifflin Harcourt.

Benitez-Quiroz, C. F., Srinivasan, R., \& Martinez, A. M. (2018). Facial color is an efficient mechanism to visually transmit emotion. Proceedings of the National Academy of Sciences, 115(14), 3581. doi:10.1073/pnas.1716084115

Chen, Z., \& Whitney, D. (2019). Tracking the affective state of unseen persons. Proceedings of the National Academy of Sciences, 116(15), 7559. doi:10.1073/pnas.1812250116

Deutsch, D. (2011). The beginning of infinity: Explanations that transform the world. London, UK: Penguin.

Ekman, P. (1999). Basic Emotions. In T. Dalgleish \& M. Power (Eds.), Handbook of Cognition and Emotion. Sussex, UK: John Wiley \& Sons.

Ekman, P., Levenson, R. W., \& Friesen, W. V. (1983). Autonomic nervous system activity distinguishes among emotions. Science, 221(4616), 1208. doi:10.1126/science.6612338

Jurist, E. (2019). Review of How Emotions Are Made: The Secret Life of the Brain. Journal of Theoretical and Philosophical Psychology, 39. doi:10.1037/teo0000098

McStay, A. (2018). Emotional AI: The rise of empathic media. London: Sage. 
Mohammad, S. M., \& Turney, P. D. (2013). Crowdsourcing a word-emotion association lexicon. Computational Intelligence, 29(3), 436-465.

Polanyi, M. (2009). The tacit dimension. Chicago: University of Chicago Press.

Sterling, P. (2012). Allostasis: A model of predictive regulation. Physiology \& Behavior, 106(1), 5-15. doi:10.1016/j.physbeh.2011.06.004

Vuong, Q. H. (2020). Reform retractions to make them more transparent. Nature, 582(149). doi.1038/d41586-020-01694-x

Vuong, Q. H., \& Napier, N. K. (2015). Acculturation and global mindsponge: an emerging market perspective. International Journal of Intercultural Relations, 49, 354-367.

Vuong, Q.-H., Bui, Q.-K., La, V.-P., Vuong, T.-T., et al. (2018). Cultural additivity: behavioural insights from the interaction of Confucianism, Buddhism and Taoism in folktales. Palgrave Communications, 4(1), 143. doi:10.1057/s41599-018-0189-2

Vuong, Q.-H., Ho, M.-T., Nguyen, H.-K. T., et al. (2020). On how religions could accidentally incite lies and violence: folktales as a cultural transmitter. Palgrave Communications, 6(1), 82. doi:10.1057/s41599-020-0442-3 
